\title{
Management of patients with chronic lymphocytic leukemia during the SARS-CoV-2 pandemic (Review)
}

\author{
ROMEO GABRIEL MIHAILA ${ }^{1,2}$ \\ ${ }^{1}$ Department of Hematology, Faculty of Medicine, Lucian Blaga University of Sibiu, 550169 Sibiu; \\ ${ }^{2}$ Department of Hematology, Emergency County Clinical Hospital, 550245 Sibiu, Romania
}

Received April 18, 2021; Accepted June 16, 2021

DOI: 10.3892/ol.2021.12897

\begin{abstract}
Oncohematological patients are prone to develop infections due to immunosuppression caused by the disease and chemo-immunotherapy. The aim of this review was to outline the details of the management of patients with chronic lymphocytic leukemia (CLL) during the severe acute respiratory syndrome coronavirus 2 (SARS-CoV-2) pandemic. Patients with CLL often exhibit inadequate humoral and cellular immune responses to various infections and vaccinations. Patients under the 'watch and wait' strategy have a lower risk of infections, including with SARS-CoV-2, compared with those undergoing therapeutic interventions, but they still have a higher risk than age-matched controls. Patients with CLL have a high risk of developing severe forms of coronavirus disease-2019 (COVID-19), particularly if they are undergoing chemo-immunotherapy. The total anti-SARS-CoV-2 antibody titer demonstrates a slower increase in patients with CLL infected with the virus, and the antibody levels tend to decrease after reaching a maximum level sooner than in healthy individuals. This leads to a late negativation of the PCR tests and a longer duration of hospitalization. In total, $\sim 1 / 3$ of patients with CLL do not develop a persistent titer of antiviral antibodies, and this is associated with the presence of hypogammaglobulinemia. It appears that patients with CLL have the worst outcomes amongst patients with malignant hemopathies and SARS-CoV-2 infection. Bruton tyrosine kinase inhibitors reduce the hyperinflammatory status of
\end{abstract}

Correspondence to: Professor Romeo Gabriel Mihaila, Department of Hematology, Faculty of Medicine, Lucian Blaga University of Sibiu, 2A Lucian Blaga Street, 550169 Sibiu, Romania E-mail: romeomihaila@yahoo.com

Abbreviations: ASH, American Society for Hematology; BTKi, Bruton Tyrosine Kinase inhibitors; CLL, chronic lymphocytic leukemia; COVID-19, coronavirus disease-2019; SARS-CoV-2, severe acute respiratory syndrome coronavirus 2

Key words: chronic lymphocytic leukemia, coronavirus disease-2019, ibrutinib, monoclonal antibodies, severe acute respiratory syndrome coronavirus 2 , venetoclax patients with CLL with COVID-19, which is accompanied by decreased levels of serum inflammatory markers, ferritin and D-dimer, and serum levels of pro-inflammatory cytokines, but they increase the risk of infections and impaired humoral immunity. An abrupt discontinuation of these may promote the rapid decompensation of CLL, which may even mimic the clinical manifestations of COVID-I9, including a significant increase in cytokine release. In conclusion, therapeutic decisions must be personalized to each patient with CLL and each at risk patient must be quarantined during the SARS-CoV-2 pandemic to reduce their risk of contraction.

\section{Contents}

1. Introduction

2. Distinct characteristics of SARS-CoV-2 infection in patients with CLL

3. Clinical experience of patients with CLL during the SARS-CoV-2 pandemic

4. Treatment of patients with CLL during the SARS-CoV-2 pandemic

5. Reducing the risk of contamination with SARS-CoV-2 in patients with CLL

6. Survey on the management of patients with CLL infected with SARS-CoV-2

7. Expert recommendations

8. Conclusions

\section{Introduction}

Chronic lymphocytic leukemia (CLL) is the most common type of leukemia in Western countries (1), with an incidence of 4.2 new cases per 100,000 individuals per year (2). The median age at diagnosis of CLL is estimated to be $\sim 72$ years (2) and several patients will have other associated diseases $(3,4)$. Patients often have inadequate humoral and cellular immune responses to various infections and vaccinations due to the induction of immunosuppression (5). In total, $70 \%$ of patients with CLL develop infections, of which $30 \%$ require hospitalization or intravenous antimicrobial treatment $(6,7)$, and this is reflected in the increased morbidity and mortality rates (6). 
The pathophysiology of complications of severe acute respiratory syndrome coronavirus 2 (SARS-CoV-2) infection is complex and not fully understood. Patients with severe forms of coronavirus disease-2019 (COVID-19) have inadequate immune responses to the virus, or otherwise the clinical manifestations are the result of an exaggerated adaptive immune response. Thus, the clinical impact of this infection on patients with CLL has not been fully elucidated (5).

The current strategy for monitoring and treating patients with CLL includes a 'watch and wait' policy that applies to several patients. Untreated patients with CLL are considered to have a lower risk of infection than those who have undergone therapeutic interventions, but a higher risk compared with age-matched controls (5). Indeed, patients with CLL have a high risk of developing a severe form of COVID-19 $(3,4)$, particularly if they are undergoing chemotherapy, due to the immunosuppressive effects of these regimens $(4,8)$ and a result of hematological malignancy. Immunomodulatory agents also increase the risk of this viral infection and alter the response to treatment (9).

Hematologists in Birmingham have published a report on the evolution of 4 treatment-naïve patients with CLL who became infected with SARS-CoV-2. Lymphocytosis increased on average by 3 -fold during infection with SARS-CoV-2, compared with the baseline levels (5). Unlike lymphopenia, which correlates with severe forms of COVID-19 and has an unfavorable evolution in the general population $(5,8)$, the clinical form was severe, and the mortality rates were high. One of the mechanisms that may explain lymphocytosis in patients with CLL infected with SARS-CoV-2 may be related to the high levels of endogenous steroids during the intense inflammatory process (5). Patients with CLL must be quarantined during the pandemic due to the risk of infection and of exhibiting a severe response, the likelihood of which is higher than that of the general population, even if they are not undergoing immunochemotherapy $(5,10)$.

An excessive immune response, similar to cytokine release syndrome, is present in severe forms of COVID-19. The patients have high serum levels of pro-inflammatory cytokines, which leads to increased morbidity and mortality (11).

Indeed, patients with CLL had the worst outcome in a group of 39 patients with malignant hemopathies and SARS-CoV-2 infection. The highest risk of death was observed in patients $\geq 70$ years of age and/or with a C-reactive protein level of $>10 \mathrm{mg} / \mathrm{dl}$ at admission (12).

Strategy research is aimed at analyzing how the therapeutic behavior of patients with CLL adapts during the SARS-CoV-2 pandemic. Scientific articles published in PubMed and Web of Science databases prior to December 31, 2020 were searched, using the terms 'chronic lymphocytic leukemia', 'COVID-19', 'ibrutinib',SARS-CoV-2', 'venetoclax'.

Mechanism of SARS-CoV-2 infection in CLL, its prophylaxis and treatment. SARS-CoV-2 enters the cell through its receptor-angiotensin-converting enzyme 2 . Thus, there is a link between inflammation, immunity, angiotensin-converting enzyme 2, and cardiovascular damage. Systemic disease triggered by SARS-CoV-2 infection is primarily due to an exaggerated and inadequate immune response (13). The inflammatory response triggered by the virus is involved in increasing the production of proinflammatory cytokines, including TNFa, IL-1 and IL-6. IL-6 is secreted by lymphocytes, macrophages, endothelial cells and fibroblasts and is involved in the regulation of many cells of the innate and adaptive immune system. Hematopoietic cells contribute to increased VEGF production, which stimulates angiogenesis, and increases T-cell differentiation to Th17 cells and Tregs, as well as B-cell activation. This inflammatory process causes damage to various organs and tissues (13).

The immune system is highly dysregulated in patients with CLL. They exhibit hypogammaglobulinemia, qualitative and quantitative B- and T-lymphocyte defects, decreased levels of CD4+ T-cells, neutropenia and innate immune dysfunction. Immune deficiency may be exacerbated by antileukemic treatment. These changes may explain the reduced ability of patients with CLL to eliminate the virus and their higher risk of contracting the infection. Instead, immunodeficiency may protect them from severe COVID-19 by limiting the excessive activation of the immune response (14).

In a group of 67 patients with malignant hemopathies, $46 \%$ did not develop anti-SARS-CoV-2 IgG antibodies after vaccination and only $23 \%$ of patients with B-lymphocyte CLL had antibodies after vaccination although almost $70 \%$ of them were not under antileukemic treatment (15).

Bruton tyrosine kinase inhibitors (BTKi) exhibit anti-inflammatory action due to their effects on both the innate immune system and platelets (14). Ibrutinib, through its immunomodulatory properties, may serve as a candidate for the treatment of clinical complications caused by excessive immune activation produced by the SARS-CoV-2 virus (13). Ibrutinib is able to reduce the production of TNFa, IL-1, IL-6, monocyte chemo-attractant protein-1, and VEGF, and it inhibits the hematopoietic cell kinase from neutrophils and macrophages involved in the regulation of cell survival and proliferation in response to IL-6 and related cytokines (16).

A recent systematic review was performed starting from the observation that BTK inhibition had a protective effect against lethal influenza-induced acute long injury in mice. The analysis included six studies with patients hospitalized for COVID-19 who had malignant lymphoproliferative diseases (some of them with CLL), and who were treated with BTK-inhibitors. This treatment, together with SARS-CoV-2 infection therapy, was associated with a lower oxygen requirement and a shorter stay in hospital (17). The therapeutic outcome of patients with CLL and COVID-19 is far from expected. General and standard agreements regarding the management and treatment of these associated diseases have only been accepted very recently (18).

The effect of CLL-specific treatments on the evolution of SARS-CoV-2 infection still requires further elucidation. The international guidelines suggest careful evaluation of the usefulness of continuing or stopping CLL treatment, especially in patients on targeted agents (19).

\section{Distinct characteristics of SARS-CoV-2 infection in patients with CLL}

It is estimated that $~ 80 \%$ of patients with CLL are asymptomatic at the time of diagnosis and that $30 \%$ of them should never be treated $(8,20)$. 
Some patients are diagnosed simultaneously with COVID-19 and CLL. This is the case of a patient with clinical manifestations suggestive of SARS-CoV-2 infection, but who had multiple lymph nodes and leukocy tosis $\left(\geq 140,000\right.$ per $\left.\mathrm{mm}^{3}\right)$ with lymphocytosis. Despite complex therapy (which included hydroxychloroquine, antibiotics, human immunoglobulins, anticoagulation, non-invasive ventilation, then intubation and mechanical ventilation), the evolution was unfavorable (8). Leukocytosis accompanied by lymphocytosis is uncommon in patients who are diagnosed with SARS-CoV-2 infection. Flow cytometry analysis was performed in this case, and it was shown that the lymphocytosis was monoclonal, and this allowed the concomitant diagnosis of CLL with SARS-CoV-2 infection (21). Patients with CLL who have newly occurring but non-specific clinical manifestations of SARS-CoV-2 infection, have indications for virological testing using PCR, followed by chest X-ray examination if the test is negative (22). A woman diagnosed simultaneously with CLL and SARS-CoV-2 infection had a high level of viremia and developed a severe form of pneumonia, although she had specific anti-IgM and anti-IgG antibodies. Antiviral therapy combined with high-dose intravenous Ig was administered, but she has had two recurrences, due to immune deficiency, during the 69-day follow-up (23).

Patients with CLL with very high leukocyte counts may, extremely rarely, experience spurious hypoxemia or pseudohypoxemia, which may create diagnostic and therapeutic difficulties (24).

A high number of regulatory T-cells and nurse-like cells, as well as dysfunction of natural killer cells and T-cells contribute to the immunodeficiency in patients with CLL (3). This may explain why patients with CLL exhibit suboptimal seroconversion following administration of vaccines $(25,26)$. The total anti-SARS-CoV-2 antibody titer increases more slowly in patients with CLL infected with this virus than in non-CLL infected patients. In addition, in patients with CLL, the antibody levels tend to decrease after reaching a maximum, while they remain at higher levels in the control group, at least until discharge. This evolution of antibodies has consequences on the negativation of the PCR tests (occurs later) and their duration of hospitalization (longer) (27). A total of 67\% of a group of 21 patients with CLL who developed SARS-CoV-2 infection and were tested for anti-SARS-CoV-2 IgG antibodies became positive for these antibodies; testing occurred at a median of 57 days (25). In this previous study, hypogammaglobulinemia of patients with CLL was negatively associated with the occurrence of anti-SARS-CoV-2 IgG. The severity of COVID-19 and ongoing anti-CLL therapy have not been associated with the development of anti-SARS-CoV-2 IgG. The study included a relatively small number of patients with CLL (same as in several other studies conducted during this pandemic) and could not analyze the dynamics of anti-SARS-CoV-2 antibodies in patients with severe COVID-19 and who died. In addition, 1/3 of CLL-tested patients infected with SARS-CoV-2 did not develop a persistent titer of antiviral antibodies (25).

Why do patients with CLL have the worst outcome inside malignant hemopathies? The monoclonality of B-cells that accumulates in human blood may be the answer. Monoclonal lymphocyte proliferation causes the dissociation of normal cell lines in the bone marrow. Certain patients with CLL may have low monocyte counts, which are able to transform into macrophages, in the blood. The reduced value or even lack of other populations of leukocytes (macrophages, granulocytes, and NK cells) may lead to an undesirable response from the immune system towards pathogens. The presence of CLL accompanied by impaired cellular and humoral immunity (mainly) (21) with secondary hypogammaglobulinemia explains the longer duration of SARS-CoV-2 infection in certain patients. Such a patient showed signs of infection for up to 70 days, and genomic and subgenomic viral shedding occurred for up to 105 days since diagnosis. During this time a continuous turnover of dominant viral variants was observed (28).

The risk of death in patients with CLL who develop symptomatic COVID-19 is estimated to be $30-33 \%(25,29)$. The factors that explain the predisposition to develop severe forms of COVID-19 in patients with CLL are: advanced age (common), immunodeficiency and inadequate serological response after various vaccinations or infections (25).

\section{Clinical experience of patients with CLL during the SARS-CoV-2 pandemic}

The majority of published articles are case presentations or refer to small groups of patients. Even multicenter studies performed on groups of oncohematological patients did not include large cohorts of patients infected with SARS-CoV-2, which is why it is difficult to draw definite conclusions.

An encouraging experience regarding SARS-CoV-2 infection in patients with CLL was published by colleagues in Barcelona. Only 4 out of 420 patients with CLL registered with the Hospital Clinic of Barcelona developed symptomatic SARS-CoV-2 infection until May 6, 2020. Their course was mild, although ferritin levels were elevated in 2 patients and D-dimers were elevated in 1 patient. Additionally, 2 of the patients had previously received CLL therapy. Recovery occurred after 4-8 days in 3 of the patients and after 24 days in 1 patient, who received experimental therapy for COVID-19 (30).

Out of 165 Spanish patients with CLL, 27\% died during acute infection with and due to SARS-CoV-2 infection. Certain patients with CLL who died were under 60 years of age and had no associated conditions (31). The mortality of Spanish patients with CLL during SARS-CoV-2 infection was higher than that of patients without neoplasms or immunosuppression hospitalized at Vall d'Hebron University Hospital. It was also higher than the mortality rate due to SARS-CoV-2 infection in Spain in May 2020, in the general population. Age, lymphocytosis, and D-dimers were associated with overall survival in patients with CLL infected with SARS-CoV-2 (24). Therefore, a more active CLL disease, which manifests as a higher number of lymphocytes, may contribute to a greater vulnerability in these patients during this infection (31).

A multicenter survey conducted in Italy on 9,930 patients with CLL studied their management in the context of the SARS-CoV-2 epidemic in April 2020. Virological testing for SARS-CoV-2 was performed in all centers with patients with CLL who had symptoms suggestive of this infection or had close contact with an infected individual. The patients were tested prior to administration of chemotherapy in only $30 \%$ of the analyzed centers (1). The triage of symptomatic patients 
and social distancing are applied in most centers. There was no significant difference between the prevalence of symptomatic SARS-CoV-2 infection in patients with CLL compared with the normal population, although the median age of the first was higher. Initiation of treatment was delayed in $79 \%$ of centers, and ongoing treatments were later performed in $24 \%$ of centers. The lack of a sufficient number of blood donors and the delay of post-therapeutic restadialization in certain centers were other problems that occurred in the management of patients with CLL. Of the 46 patients with CLL who developed symptomatic SARS-CoV-2 infection, $30.4 \%$ died. The rate was higher than that of the Italian general population aged between 70-79 years (1).

A total of 23 of the 2,902 patients with CLL at 6 different hematology centers in Lombardy developed COVID-19, which was documented virologically (32). All patients with CLL with COVID-19 had pneumonia and 2 required oro-tracheal intubation. In total, 1 patient with CLL with comorbidities, treated with ibrutinib, died. The authors mention that it cannot be ruled out that other patients with CLL also had SARS-CoV-2 infection, but were asymptomatic or oligosymptomatic, and was thus not reported by patients. They also suggest that ibrutinib could have prevented the progression to severe forms of COVID-19, due to its immunomodulatory effects. In addition, ibrutinib inhibits IL-2-inducible T-cell kinase activity; thus, it is involved in restoring the Th1/Th2 balance, specifically, an imbalance in the Th1 phenotype in patients with CLL. In the severe forms of SARS-CoV-2 infection, the level of pro-inflammatory cytokines, such as IL-6, increases and the proportion of Th17 cells increases compared with $\mathrm{CD} 4^{+}$and CD8 ${ }^{+}$lymphocytes. IL- 6 stimulates the differentiation of Th17 cells into Th0, which serve an essential role in the release of cytokines (32-34).

A multicenter analysis that included 198 patients with CLL who developed a symptomatic form of SARS-CoV-2 infection showed that the disease was more severe in these patients compared with the general population. The case fatality rate of hospitalized patients was 37\% (35). Such a Chinese patient with uncontrolled CLL and several associated diseases developed a severe form of COVID-19 that led to death (36).

Indeed, patients with CLL infected with SARS-CoV-2 are predisposed to develop severe forms of COVID-19 (79\% of 190 patients included in a European study). They required oxygen therapy and/or admission to intensive care (14). Patients aged $\geq 65$ years were more likely to develop more severe forms of the disease, but age and comorbidities did not influence mortality. Deaths were more common in patients with severe COVID-19 (36.4 vs. $2.6 \%$ in those with mild disease). CLL treatment seemed to have a protective role (14).

Experts recommend repeated testing for SARS-CoV-2 infection using PCR tests in patients who have undergone an allogeneic stem cell transplant if the first test was negative, due to their high rate of false-negative tests. Symptomatology, pulmonary imaging, and testing for the presence of the virus in broncho-alveolar lavage may help increase the detection of infected patients (37).

In such a case, a female patient who received a haploidentical allogeneic stem cell transplant for B-cell acute lymphoblastic leukemia 157 days ago, and who showed symptoms suggestive of SARS-CoV-2, came back with both nasal and nasopharyngeal PCR swab tests that were negative for this viral infection. It should be noted that she was receiving corticosteroid therapy and weekly administration of rituximab for a chronic graft vs. host disease of the skin and mouth. Only PCR/CRISPR technology applied to body fluid samples helped to detect the viral infection (37). Intravenous immunoglobulin (administered due to the presence of hypogammaglobulinemia), antibiotic therapy, oxygen therapy and transfusion with COVID-19 convalescent plasma had improved clinical and biological evolution. A male patient with high-risk acute myeloid leukemia received an allogeneic stem cell transplant 319 days ago. He was under treatment with ibrutinib, prednisone and rituximab for a chronic skin and lung conditions when he presented with dyspnea, cough, and a worsening skin rash. A CT chest detected opacities suggestive of COVID-19, but testing of nasal exudate by PCR was negative. Only PCR/CRISPR, that was performed on a blood sample, allowed the detection of SARS-CoV-2 RNA (37).

The number of T-lymphocytes is reduced in stem cell transplant recipients. Graft vs. host disease therapy contributes to a further decrease in their levels. The decrease in T-lymphocytes was correlated with the risk of developing a severe form of COVID-19 and with the risk of death during hospitalization (38).

\section{Treatment of patients with CLL during the SARS-CoV-2 pandemic}

Anti-leukemic treatments. In patients with CLL with controlled disease who develop an infectious respiratory disease, discontinuation of anti-leukemic therapy is not indicated unless it produces significant immunosuppression (39).

Anti-leukemic treatments can increase an individual's susceptibility to infections, which substantially increase the morbidity and mortality of patients with CLL. Furthermore, SARS-CoV-2 infection may recur in patients with CLL (19). Certain patients with CLL exhibit longer COVID-19 infections. Such a patient treated with chemoimmunotherapy had fever and severe viral pneumonia. The infection lasted 9 weeks, during which he was treated with remdesivir given in two 10-day courses, and in these periods the fever disappeared, but it reappeared afterwards (40).

Patients on recent or undergoing treatment for CLL from the study led by Scarfò et al (14) (a joint study by the European Research Initiative on CLL) more frequently presented with mild forms of COVID-19, than those with severe forms of the disease who were monitored according to the watch and wait strategy. The number of patients undergoing chemotherapy was so limited compared with those treated with BTKi that no conclusions could be drawn regarding the efficacy of different types of anti-CLL therapies during the SARS-CoV-2 pandemic (14). This is found in most hematology centers, which have updated their therapeutic regimens according to the latest guidelines.

The hospitalized patients with CLL have a high risk of mortality ( $1 / 3$ higher), regardless of whether or not they were treated before the onset of SARS-CoV-2 infection or disease phase, according to a multicenter study that included 198 symptomatic COVID-19 patients with CLL. It should be noted that $76 \%$ of those treated received BKTi, which were 
stopped during the course COVID-19 infection in the majority of patients with CLL (29).

Superinfection of SARS-CoV-2 infected patients with various bacteria or fungi appears to increase the risk of death. A patient with CLL infected with SARS-CoV-2 was co-infected with parainfluenza due to the immunosuppression (41).

Treatment with convalescent plasma. The early decrease in B-lymphocyte counts observed in patients with COVID reduces their ability to produce antibodies and justifies the use of COVID-19 convalescent plasma transfusions, which introduces beneficial neutralizing antibodies (42). It has been suggested that convalescent plasma is more effective if administered in the first 14 days after the onset of the disease (43).

Treatment with Bruton Tyrosine Kinase inhibitors. BTK is actively involved in B-cell development and functionality, including antibody production (32). In addition, BTK is expressed in various other cells, such as granulocytes, macrophages, monocytes, and dendritic cells (14).

It was observed that BTK activity is increased in blood monocytes obtained from patients with a severe form of COVID-19 (44). Acting on certain key transcription factors, BTK is involved in regulating macrophage polarization (45).

The half-life of ibrutinib and acalabrutinib in patients without renal dysfunction is 4-7 h, but these drugs bind covalently to BTK; pathway re-activation depends on de novo enzymatic synthesis, and this occurs at extremely variable rates from one patient to another. Therefore, BTK inhibition may persist for a long time in some patients (11).

It has previously been shown that ibrutinib is able to reduce the levels of proinflammatory cytokines in the lungs, lung damage and animal deaths (46). Thus, the hyperinflammatory status of COVID-19 also decreases in patients treated with BTKi (45), such as ibrutinib, acalabrutinib and zanubrutinib (11). It also includes a reduction in serum levels of pro-inflammatory cytokines, the levels of which are increased substantially in severe forms of COVID-19 (11).

Amongst the inflammatory markers, ferritin and D-dimer were present at lower levels in patients with CLL during treatment with BTKi at the time of SARS-CoV-2 infection compared with patients who received other treatments, and this may be explained by the immunomodulatory effect of BTKi (31). Overall survival was the same in patients with CLL, regardless of whether they received treatment during the SARS-CoV-2 infection period. This observation also includes the patients treated with BTKi, whose mortality rate did not differ from that of those to whom the 'watch and wait' strategy was applied (31).

However, treatment with BTKi increases the risk of infections (45), particularly viral infections in patients with CLL (11), and impairs humoral immunity (45), including the response to SARS-CoV-2 virus and the protection against possible subsequent bacterial infections (9), which would suggest the need to discontinue administration of BTKi. However, the decrease in hyperinflammatory status is more important. In addition, stopping the administration of BTKi may contribute to the rapid progression of a lymphoproliferative disease (45). Indeed, an abrupt discontinuation of B-cell receptor kinase inhibitors may promote rapid decompensation of CLL, which may even mimic the clinical manifestations of COVID-19 (9). In addition, abrupt discontinuation of ibrutinib leads to significant cytokine release $(11,47)$, and long-term BTKi therapy contributes significantly to the recovery of humoral immune function, followed by decreased infection rates $(11,48)$.

Studies have found that the use of BTKi is able to reduce the need for mechanical ventilation and can reduce the mortality rates during hospitalization of patients with severe forms of disease produced by SARS-CoV-2 infection. This medication seems promising for decreasing the amplitude of the immune response triggered by infection and patient mortality (44). Another study also found that ibrutinib contributed to a shorter hospital stay in patients with CLL and a severe form of COVID-19 compared with those treated with other drugs or not treated at all (14). Therefore, the decision to continue BTKi therapy should take into account the individual's risk of impaired humoral immunity (9).

An American study presented the evolution of 8 patients with CLL who were treated with BTKi infected with SARS-CoV-2: 2 of the patients, in whom BTKi was stopped, developed severe forms of COVID-19, and the others presented with a mild-to-moderate form of the disease. The 2 patients who continued ibrutinib treatment had shorter hospitalization periods, required minimal oxygen, and recovered from the infection (11). No significant adverse effects were observed in the patients with CLL who were treated with BTKi, but they were not treated with a full-dose of the anti-coagulant, which may be necessary in patients with severe forms of COVID-19. BTKi therapy should be used with caution in patients with such forms (11).

Venetoclax treatment. It has been suggested that chemotherapeutic regimens that include venetoclax would decrease immunosuppression of patients with CLL (49). In a multicenter phase 3 trial (using treatment-naive patients), 7 patients with CLL developed SARS-CoV-2 infection; 1 patient was 22 months after the end of therapy, and the other 6 were included in the experimental arms of chemoimmunotherapy that included venetoclax. Of these patients, 6 were hospitalized, and 2 of them required intensive care; 2 patients died, one after prolonged mechanical ventilation and the other, who refused intensive care treatment, following the best supportive care. Most patients had at least one episode of neutropenia during the clinical trial and some even had repeated infections. The explanation for the immune suppression is multifactorial: Low pre-therapeutic levels of serum $\operatorname{IgG}$ in 6 of the patients, low levels of TH and TS lymphocytes (4), and functional deficits of T-lymphocytes $(4,50)$. The death rate was comparable to that of patients with CLL treated with BTKi (4). The patients treated with venetoclax-based chemoimmunotherapy had increased rates of SARS-CoV-2 infection and required increased hospitalization, but most patients recovered from COVID-19 (4).

\section{Reducing the risk of contamination with SARS-CoV-2 in patients with CLL}

Unanimously accepted measures to reduce the risk of contamination of patients with CLL and SARS-CoV-2 
include: Minimizing non-essential hospital visits and replacing them, whenever possible, with telehealth sessions; reducing the time patients with CLL spend in infusion wards and other clinical areas; reducing the use of therapies that induce strong immunosuppression; reducing healthcare utilization during this pandemic; and personalization of treatment taking into account the disease risk, the degree of immunosuppression, the prevalence and transmission rate of the virus in the region and the existing healthcare availability (51).

Several practical measures for the application of telemedicine have been adopted in a cancer center in Madrid, to reduce the number of visits of patients with CLL in the hospital and reduce the risk of SARS-CoV-2 contamination. A portal through which patients could communicate with the medical team was created; questionnaires regarding the clinical manifestations of CLL and COVID-19, adverse events, and quality of life were developed and were to be completed periodically by patients at home. Access had been created for all patients to a patient-reported outcome program in order to provide data on patients' health and patient-centered care; the pharmacy department developed a home-delivery system through which patients with CLL receive prescriptions at home. The authors appreciate that the questionnaires can replace appropriate clinical examination, and in case of reporting symptoms or signs suggestive of a progression of CLL or SARS-CoV-2 infection, the patient is invited to the hospital for consultation and virological testing (52).

\section{Survey on the management of patients with CLL infected with SARS-CoV-2}

Several CLL experts from different countries have completed a survey on the management of patients with CLL infected with SARS-CoV-2. Most respondents considered that the prevention of this infection and social distancing can be achieved according to the recommendations of the World Health Organization, by obtaining the necessary food and medicine, but by giving up those important activities that expose an individual to an increase chance of contact with other individuals, some of which can be infected (9). Regarding the use of masks, experts opted for the surgical masks, as N-95 masks existed in limited quantities (in April 2020) and thus should be kept for healthcare workers, who are at increased risk of coming in contact with patients with COVID-19. Most experts considered that not all patients with CLL should be tested (only symptomatic ones) as long as the test supply is limited. Most respondents maintained that only patients with CLL who call and state that they have symptoms should be tested, even if the testing possibilities are unlimited. The majority of respondents opted to discontinue CLL therapy in outpatients with mild symptoms $(60.5 \%)$ or to continue it if the clinical situation requires it (25.5\%). However, $44 \%$ of respondents thought that BTKi therapy should be continued. More respondents opted to continue BTKi therapy in the COVID-19 inpatients (but not in intensive care units) compared with other chemotherapeutics (32.5 vs. 4\%). Most experts $(72 \%)$ have not advocated for the administration of intravenous Ig administration in patients with CLL with this viral infection. However, this decision does not include the patients with secondary pneumonia and those with severe hypogammaglobulinemia (9).

\section{Expert recommendations}

The American Society for Hematology recommends testing patients with CLL with mild clinical manifestations of SARS-CoV-2 infection, based on test and treatment availability and the need to isolate positive cases from other patients. With the exception of patients present in the clinic, certain doctors may only test symptomatic patients who require treatment, due to the limited number of tests available and the risk of spreading the disease. Other practitioners test aggressively to detect infection with SARS-CoV-2 and other respiratory viruses, although patients may have minor clinical manifestations due to the risk of other pathogens and to isolate any subject who may transmit a virus. However, patients with more severe symptoms should be tested virologically.

It is common in medical practice for treatment to be delayed, if possible.

If patients require immediate initiation of therapy, it is advisable to offer them the best therapy scheme, which is adapted to the patient's distinct characteristics. When there are therapeutic options, it is preferred to use drugs which can be administered in an outpatient setting and requires as few controls as possible in the clinic and laboratory tests. It is advisable to avoid monoclonal antibodies, especially in combination with targeted agents. It is also recommended to avoid initiating venetoclax treatment, as it requires numerous and extensive visits to the clinic and laboratory examinations. Venetoclax will only be given to a specific patient if it is considered the most appropriate medicine for them.

If patients with CLL are not infected with SARS-CoV-2, it is recommended to continue the administration of intravenous Ig only if they have had hypogammaglobulinemia in their personal history and active or numerous, severe infections. Infusions should be administered less frequently, and the target level of IgG should be $400-500 \mathrm{mg} / \mathrm{dl}$. If patients with CLL have COVID-19, they can continue intravenous Ig therapy. The decision should take into account the increased thromboembolic risk of patients with COVID-19, and patients should be evaluated for risk/benefit ratio, and closely monitored for possible thromboembolic events.

For monitoring, it is recommended that stable patients with CLL use laboratories closest to their home and telemedicine to minimize the number visits to the clinic. The majority of patients with CLL should continue ongoing therapies, except for monoclonal antibodies and intravenous Ig $(7,48)$.

Patients infected with SARS-CoV-2. It is not recommended to change the CLL therapy if outpatients have mild symptoms of SARS-CoV-2 infection. The decision to change CLL treatment in patients with more severe clinical manifestations must take into account, on the one hand, the aggressiveness of CLL and the presence of frequent infections in the individual's personal history and, on the other hand, the potential risk of more serious complications of COVID-19. There is not yet sufficient clinical evidence to allow a different approach for the classes of chemotherapeutics 
used for CLL therapy. The continuation or cessation of the treatment is established according to the particularities of each case. There is a consensus to stop the administration of monoclonal antibodies in patients with COVID-19. Discontinuation of therapy with a B-cell receptor signaling inhibitor may promote the onset of a CLL flare and the release of pro-inflammatory cytokines, which may mimic the clinical and laboratory manifestations of COVID-19. Resumption of therapy with the B-cell receptor signaling inhibitor is generally accompanied by the relatively rapid disappearance of clinical manifestations $(7,53)$.

The European Hematology Association has taken over and disseminated the recommendations made by $\mathrm{ASH}$ for the therapy of patients with CLL during the SARS-CoV-2 pandemic.

ASH has developed a COVID-19 registry for oncohematological patients, which may assist in improving the treatment of these patients (including those with CLL), as experience and knowledge regarding the therapy of this viral infection and the evolution of oncohematological patients under various therapies will be enriched. It is important to learn from experience to optimize the treatment of the patients (7).

\section{Conclusions}

Patients with CLL have a high risk of developing a severe form of COVID-19, due to their humoral and cellular immune deficiency. SARS-CoV-2 can worsen the health condition of patients with CLL, particularly due to the progressive reduction/lack of the population of cells required in the fight against pathogens; immuno-chemotherapy can aggravate this immunodeficiency.

Therefore, it is recommended to reduce clinical visits as much as possible, as well as waiting times and duration of infusions. Ideally, patients should quarantine during the SARS-CoV-2 pandemic to reduce their risk of contracting this virus.

If the patient requires immediate initiation of therapy, it is advisable to adapt the therapeutic behavior to the particularities of each patient. It is indicated to avoid monoclonal antibodies, especially in combination with targeted agents. Chemotherapeutic regimens that can be applied at home are preferred and monitoring of patients should be performed by telemedicine (by completing questionnaires and telephone discussions) and using laboratories located closest to the patients' homes.

It is not recommended to change CLL therapy if outpatients have mild symptoms of SARS-CoV-2 infection. CLL treatment in patients with more severe clinical manifestations depends on the aggressiveness of CLL and the presence of frequent infections in personal history, but also on the potential risk of more serious complications of COVID-19.

BTKi are able to decrease the hyperinflammatory status and the serum levels of pro-inflammatory cytokines in patients with CLL with severe forms of COVID-19. However, they increase the risk of infections and impaired humoral immunity. Abrupt discontinuation of BTKi may contribute to the rapid decompensation of CLL, which may even mimic the clinical manifestations of COVID-I9, and the onset of a significant cytokine release.
The SARS-CoV-2 pandemic is a challenge not only for patients but also for physicians. The experience gained every day regarding therapy of patients with CLL in the current pandemic and the multitude of articles that are published each month will allow continuous improvement of the management of patients with CLL.

\section{Acknowledgements}

Not applicable.

\section{Funding}

No funding was received.

\section{Availability of data and materials}

Not applicable.

\section{Authors' contributions}

RGM wrote and revised the manuscript. Data authentication is not applicable. RGM read and approved the final manuscript.

\section{Ethics approval and consent to participate}

Not applicable.

\section{Patient consent for publication}

Not applicable.

\section{Competing interests}

The author declares that they have no competing interests.

\section{References}

1. Cuneo A, Scarfò L, Reda G, Varettoni M, Quaglia FM, Marchetti M, De Paoli L, Re F, Pietrasanta D, Rigolin GM, et al: Chronic lymphocytic leukemia management in Italy during the COVID-19 pandemic: A Campus CLL report. Blood 136: 763-766, 2020.

2. Eichhorst B, Robak T, Montserrat E, Ghia P, Niemann CU, Kater AP, Gregor M, Cymbalista F, Buske C, Hillmen P, et al: Chronic lymphocytic leukaemia: ESMO clinical practice guidelines for diagnosis, treatment and follow-up. Ann Oncol 32: 23-33, 2021.

3. Riches JC and Gribben JG: Understanding the immunodeficiency in chronic lymphocytic leukemia: Potential clinical implications. Hematol Oncol Clin North Am 27: 207-235, 2013.

4. Fürstenau M, Langerbeins P, De Silva N, Fink AM, Robrecht S, von Tresckow J, Simon F, Hohloch K, Droogendijk J, van der Klift M, et al: COVID-19 among fit patients with CLL treated with venetoclax-based combinations. Leukemia 34: 2225-2229, 2020

5. Paneesha S, Pratt G, Parry H and Moss P: Covid-19 infection in therapy-naive patients with B-cell chronic lymphocytic leukemia. Leuk Res 93: 106366, 2020.

6. Williams AM, Baran AM, Meacham PJ, Feldman MM, Valencia HE, Newsom-Stewart C, Gupta N, Janelsins MC, Barr PM and Zent CS: Analysis of the risk of infection in patients with chronic lymphocytic leukemia in the era of novel therapies. Leuk Lymphoma 59: 625-632, 2018.

7. Sehn LH, Kuruvilla P, Christofides A and Stakiw J: Management of chronic lymphocytic leukemia in Canada during the coronavirus pandemic. Curr Oncol 27: e332-e335, 2020. 
8. Charra B, Ellouadghiri A, Magramane A, Kebbou T, Damaan K, Maghfour A, Seddiki K and Ezzouine H: COVID-19 and fortuitous discovery of chronic lymphocytic leukemia: Biological findings and therapeutic challenges. Pan Afr Med J 36: 286, 2020.

9. Koffman B, Mato A, Byrd JC, Danilov A, Hedrick B, Ujjani C, Roeker L, Stephens DM, Davids MS, Pagel JM and Shadman M: Management of CLL patients early in the COVID-19 pandemic: An international survey of CLL experts. Am J Hematol 95: E199-E203, 2020.

10. Strati P and Shanafelt TD: Monoclonal B-cell lymphocytosis and early-stage chronic lymphocytic leukemia: Diagnosis, natural history, and risk stratification. Blood 126: 454-462, 2015.

11. Thibaud S, Tremblay D, Bhalla S, Zimmerman B, Sigel K and Gabrilove J: Protective role of Bruton tyrosine kinase inhibitors in patients with chronic lymphocytic leukaemia and COVID-19. Br J Haematol 190: e73-e76, 2020.

12. Sanchez-Pina JM, Rodríguez Rodriguez M, Castro Quismondo N, Gil Manso R, Colmenares R, Gil Alos D, Paciello ML, Zafra D, Garcia-Sanchez C, Villegas C, et al: Clinical course and risk factors for mortality from COVID-19 in patients with haematological malignancies. Eur J Haematol 105: 597-607, 2020

13. Molina-Cerrillo J, Marquet-Palomanes J, Alonso-Gordoa T, López-Jiménez J and Grande E: May ibrutinib have activity in respiratory complications by SARS-CoV-2? Clinical experience in a patient with chronic lymphocytic leukemia. Healthcare (Basel) 9: 78, 2021.

14. Scarfò L, Chatzikonstantinou T, Rigolin GM, Quaresmini G, Motta M, Vitale C, Garcia-Marco JA, Hernandez-Rivas JÁ, Mirás F, Baile M, et al: COVID-19 severity and mortality in patients with chronic lymphocytic leukemia: A joint study by ERIC, the European research initiative on CLL, and CLL Campus. Leukemia 34: 2354-2363, 2020.

15. Agha M, Blake M, Chilleo C, Wells A and Haidar G: Suboptimal response to COVID-19 mRNA vaccines in hematologic malignancies patients. medRxiv: doi: 10.1101/2021.04.06.21254949 (Preprint).

16. Poh AR, O'Donoghue RJ and Ernst M: Hematopoietic cell kinase $(\mathrm{HCK})$ as a therapeutic target in immune and cancer cells. Oncotarget 6: 15752-15771, 2015.

17. Stack M, Sacco K, Castagnoli R, Livinski AA, Notarangelo LD and Lionakis MS: BTK inhibitors for severe acute respiratory syndrome coronavirus 2 (SARS-CoV-2): A systematic review. Res Sq: doi: 10.21203/rs.3.rs-319342/v1 (Preprint)

18. Roosta Y, Behzadi F, Askari E, Raeisi M, Danandeh Mehr A and Nouri-Vaskeh M: Concurrent chronic lymphocytic leukemia and COVID-19: A comprehensive review of epidemiological, diagnostic, and therapeutic challenges. Leuk Res Rep 15: 100239, 2021.

19. Rossi D, Shadman M, Condoluci A, Brown JR, Byrd JC Gaidano G, Hallek M, Hillmen P, Mato A, Montserrat E and Ghia P: How we manage patients with chronic lymphocytic leukemia during the SARS-CoV-2 pandemic. Hemasphere 4 : e432, 2020

20. Jacque $\mathrm{N}$ and Leblond V: La leucémie lymphoïde chronique: Mise au point. Presse Med 48: 807-815, 2019.

21. Ali E, Badawi M, Abdelmahmuod E, Kohla S and Yassin MA: Chronic lymphocytic leukemia concomitant with COVID 19: A case report. Am J Case Rep 21: e926062, 2020

22. Ting FI, Sacdalan DB, Cortez JL, Pacana MAD and Jimeno C: COVID-19 in cancer patients can be challenging to screen in a resource limited setting. Cancer Treat Res Commun 25: 100214, 2020.

23. Ye X, Xiao X, Li B, Zhu W, Li Y, Wu J, Huang X, Jin J, Chen D, Jin J and Huang J: Low humoral immune response and ineffective clearance of SARS-Cov-2 in a COVID-19 patient with CLL during a 69-day follow-up. Front Oncol 10: $1272,2020$.

24. Alves Barbosa O, Guimarães Andrade T, de Almeida Sousa MD and Correia JW: COVID-19 in a patient with chronic lymphocytic leukaemia with pseudohypoxemia. Eur J Case Rep Intern Med 7: 001763,2020.

25. Roeker LE, Knorr DA, Pessin MS, Ramanathan LV, Thompson MC, Leslie LA, Zelenetz AD and Mato AR: Anti-SARS-CoV-2 antibody response in patients with chronic lymphocytic leukemia. Leukemia 34: 3047-3049, 2020.

26. Sinisalo M, Aittoniemi J, Käyhty $\mathrm{H}$ and Vilpo J: Vaccination against infections in chronic lymphocytic leukemia. Leuk Lymphoma 44: 649-652, 2003
27. Favresse J, Eucher C, Elsen M, Graux C, Goebels P, Laffineur K, Nicolas JB, Dogné JM and Douxfils J: Unexpected kinetics of anti-SARS-CoV-2 total antibodies in two patients with chronic lymphocytic leukemia. Br J Haematol 190: e187-e189, 2020.

28. Avanzato VA, Matson MJ, Seifert SN, Pryce R, Williamson BN, Anzick SL, Barbian K, Judson SD, Fischer ER, Martens C, et al: Case study: Prolonged infectious SARS-CoV-2 shedding from an asymptomatic immunocompromised individual with cancer. Cell 183: 1901-1912.e9, 2020.

29. Mato AR, Roeker LE, Lamanna N, Allan JN, Leslie L, Pagel JM, Patel K, Osterborg A, Wojenski D, Kamdar M, et al: Outcomes of COVID-19 in patients with CLL: A multicenter international experience. Blood 136: 1134-1143, 2020.

30. Baumann T, Delgado J and Montserrat E: CLL and COVID-19 at the hospital clinic of Barcelona: An interim report. Leukemia 34: 1954-1956, 2020.

31. Muntañola A, Villacampa G, Hernández-Rivas JÁ, Alonso R, Mirás F, Osorio S, Baile M, Baltasar P, López Jiménez J, Hernandez-Rodriguez I, et al: Clinical characteristics and outcome of SARS-CoV-2 infection in admitted patients with chronic lymphocytic leukemia from a single European country. Exp Hematol Oncol 9: 37, 2020.

32. Reda G, Noto A, Cassin R, Zamprogna G, Borella C, Scarfò L, Farina L, Molteni A, Ghia P, Tedeschi A, et al: Reply to 'CLL and COVID-19 at the hospital clinic of Barcelona: An interim report' analysis of six hematological centers in Lombardy: On behalf of CLL commission of Lombardy hematology network (REL). Leukemia 34: 2531-2532, 2020.

33. Zhang C, Wu Z, Li JW, Zhao H and Wang GQ: The cytokine release syndrome (CRS) of severe COVID-19 and Interleukin-6 receptor (IL-6R) antagonist Tocilizumab may be the key to reduce the mortality. Int J Antimicrob Agents 55: 105954, 2020.

34. Miossec P and Kolls JK: Targeting IL-17 and TH17 cells in chronic inflammation. Nat Rev Drug Discov 11: 763-776, 2012.

35. Montserrat E: When CLL meets COVID-19. Blood 136 1115-1116, 2020.

36. Song SH, Chen TL, Deng LP, Zhang YX, Mo PZ, Gao SC, $\mathrm{Hu}$ WJ, Xiong Y and Ma ZY: Clinical characteristics of four cancer patients with SARS-CoV-2 infection in Wuhan, China. Infect Dis Poverty 9: 82, 2020.

37. Niu A, McDougal A, Ning B, Safa F, Luk A, Mushatt DM, Nachabe A, Zwezdaryk KJ, Robinson J, Peterson T, et al: COVID-19 in allogeneic stem cell transplant: High false-negative probability and role of CRISPR and convalescent plasma. Bone Marrow Transplant 55: 2354-2356, 2020.

38. Xu B, Fan CY, Wang AL, Zou YL, Yu YH, He C, Xia WG, Zhang JX and Miao Q: Suppressed T cell-mediated immunity in patients with COVID-19: A clinical retrospective study in Wuhan, China. J Infect 81: e51-e60, 2020.

39. von Lilienfeld-Toal M, Vehreschild JJ, Cornely O, Pagano L and Compagno F; EHA Infectious Disease Scientific Working Group, Hirsch HH: Frequently asked questions regarding SARS-CoV-2 in cancer patients-recommendations for clinicians caring for patients with malignant diseases. Leukemia 34: 1487-1494, 2020

40. Helleberg M, Niemann CU, Moestrup KS, Kirk O, Lebech AM, Lane C and Lundgren J: Persistent COVID-19 in an immunocompromised patient temporarily responsive to two courses of remdesivir therapy. J Infect Dis 222: 1103-1107, 2020

41. Langerbeins P, Fürstenau M, Gruell H, Klein F, Persigehl T, Rybniker J, Seeger-Nukpezah T, Kochanek M, Hallek M, Eichhorst B, et al: COVID-19 complicated by parainfluenza co-infection in a patient with chronic lymphocytic leukemia. Eur J Haematol 105: 508-511, 2020.

42. Lin L, Lu L, Cao W and Li T: Hypothesis for potential pathogenesis of SARS-CoV-2 infection-a review of immune changes in patients with viral pneumonia. Emerg Microbes Infect 9: 727-732, 2020

43. Cheng Y, Wong R, Soo YO, Wong WS, Lee CK, Ng MH, Chan P, Wong KC, Leung CB and Cheng G: Use of convalescent plasma therapy in SARS patients in Hong Kong. Eur J Clin Microbiol Infect Dis 24: 44-46, 2005.

44. Rada M, Qusairy Z, Massip-Salcedo M and Macip S: Relevance of the bruton tyrosine kinase (BTK) as a target for COVID-19 therapy. Mol Cancer Res 19: 549-554, 2020.

45. Chong EA, Roeker LE, Shadman M, Davids MS, Schuster SJ and Mato AR: BTK Inhibitors in cancer patients with COVID-19: 'The winner will be the one who controls that chaos' (Napoleon Bonaparte). Clin Cancer Res 26: 3514-3516, 2020. 
46. Treon SP, Castillo JJ, Skarbnik AP, Soumerai JD, Ghobrial IM Guerrera ML, Meid K and Yang G: The BTK inhibitor ibrutinib may protect against pulmonary injury in COVID-19-infected patients. Blood 135: 1912-1915, 2020.

47. Castillo JJ, Gustine JN, Meid K, Dubeau T, Severns P and Treon SP: Ibrutinib withdrawal symptoms in patients with Waldenström macroglobulinemia. Haematologica 103: e307-e310, 2018.

48. Sun C, Tian X, Lee YS, Gunti S, Lipsky A, Herman SE, Salem D, Stetler-Stevenson M, Yuan C, Kardava L, et al: Partial reconstitution of humoral immunity and fewer infections in patients with chronic lymphocytic leukemia treated with ibrutinib. Blood 126: 2213-2219, 2015.

49. de Weerdt I, Hofland T, de Boer R, Dobber JA, Dubois J, van Nieuwenhuize D, Mobasher M, de Boer F, Hoogendoorn M, Velders GA, et al: Distinct immune composition in lymph node and peripheral blood of CLL patients is reshaped during venetoclax treatment. Blood Adv 3: 2642-2652, 2019.

50. Riches JC, Davies JK, McClanahan F, Fatah R, Iqbal S, Agrawal S, Ramsay AG and Gribben JG: T cells from CLL patients exhibit features of T-cell exhaustion but retain capacity for cytokine production. Blood 121: 1612-1621, 2013.
51. Di Ciaccio P, McCaughan G, Trotman J, Ho PJ, Cheah CY, Gangatharan S, Wight J, Ku MH, Quach H, Gasiorowski R, et al: Australian and New Zealand consensus statement on the management of lymphoma, chronic lymphocytic leukaemia and myeloma during the COVID-19 pandemic. Intern Med J 50: 667-679, 2020

52. Cordoba R: Bringing hospital care closer to patients amidst COVID-19. Lancet Haematol 7: e637, 2020.

53. American Society of Hematology (ASH): COVID-19 and CLL: Frequently asked questions. ASH, Washington, DC, 2020 http://www.hematology.org/covid-19/covid-19-and-cll. Last updated February 2, 2021

cc) (i) (9) This work is licensed under a Creative Commons CY AC ND Attribution-NonCommercial-NoDerivatives 4.0 International (CC BY-NC-ND 4.0) License. 